\title{
A novel artificial neural network-based algorithm for detecting vascular occlusive diseases in retinal images
}

\author{
L.R.A.B. Jayaratne ${ }^{1 *}$, V.H.W. Dissanayake ${ }^{2}$ \\ 1 National Hospital of Sri Lanka, Colombo, Sri Lanka \\ 2 Postgraduate Institute of Medicine, University of Colombo, Sri Lanka \\ * jayaratne.Irab2017@pgim.cmb.ac.lk \\ iD https://orcid.org/0000-0002-0791-3334
}

\begin{abstract}
Introduction: Central retinal vascular occlusions cause sudden painless complete blindness of one eye or partial visual loss, depending on the retinal vessel involved. These acute conditions may occur due to thrombosis, diabetic retinopathy, hypertensive retinopathy, glaucoma or due to other systemic conditions like connective tissue disorders. Central retinal artery occlusion (CRAO) is considered analogous to a cerebral stroke and it is an ophthalmological emergency. Central retinal vein occlusion (CRVO) is one of the most common causes of visual loss worldwide and the second most common cause of blindness due to retinal vascular disorders after diabetic retinopathy.
\end{abstract}

Artificial intelligence (Al) based methodologies have been used in automated diagnosis support systems in detecting many ophthalmological conditions like diabetic retinopathy, retinal micro aneurisms, and retinal haemorrhages.

Methods: The developed algorithm consists of modules for, image pre-processing, extraction of the areas of interest, feature extraction, feature presentation and image classification. A feed-forward back-propagation multilayer artificial neural network (ANN), with 27 input layer neurones, three output layer neurones and 81 first hidden layer and nine second hidden layer neurones, was used as the image classifier. This methodology was trained with 90 retinal images.

Results: The percentage accuracy of the developed ANN-based ophthalmological image classification algorithm is $97.8 \%$ when it is trained to diagnose CRAO and CRVO. The sensitivity is $98.3 \%$, and the specificity is $100 \%$.

Conclusions: This study presents the first Al-based approach in automatic detection of both CRAO and CRVO. Once this algorithm is trained to minimise false negatives, it can be used as an automated diagnosis support system in ophthalmological screening programs.

Keywords: Artificial Intelligence, Artificial Neural Network, Diagnosis Support System, Central retinal artery occlusion, Central retinal vein occlusion 